\title{
Diseño de un proceso para la obtención de pectina en medio ácido a partir de la cáscara de papa (Solanum tuberosum)
}

Design of a process for the obtaining of pectin in acid medium from the potato husk (Solanum tuberosum)

Erika Gabriela Toapanta. ${ }^{1}$, Sonia Vallejo Abarca . ${ }^{2}$, Marlene Jaqueline García ${ }^{3}$ \& Edmundo Caluña Sánchez ${ }^{4}$

Recibido: 27-03-2019 / Revisado: 27-04-2019 /Aceptado: 25-05-2019/ Publicado: 05-06-2019

\begin{abstract}
.
DOI: https://doi.org/10.33262/cienciadigital.v3i2.6.522
\end{abstract}

The present research work proposes for designing the process for obtaining pectin from potato husks (Solanum tuberosum) at laboratory Analytical Chemistry at Escuela Superior Politécnica de Chimborazo. it carried out several processes, starting with the pre-treatment of raw material to which the physicochemical characteristics were determined with a bromatological analysis where the following values were obtained: Fiber $4.37 \%$, Humidity $83.72 \%$, Raw material used was the (Variety of potatoSuperchola). Then, acid hydrolysis was performed where an experimental designTaguchi was used, which allowed to interact the most important variables from this process: $\mathrm{pH}$, temperature and hydrolysis time found in this way the best extraction conditions: $\mathrm{pH} 1.5$, extraction time $60^{\prime}$ and extraction temperature $90{ }^{\circ} \mathrm{C}$; with these parameters, a yield of $14.34 \%$ was obtained. For pectin precipitation $96^{0}$ ethanol was used with a ratio of $40 \%$ for the hydrolysis liquid and the drying was carried out a temperature of $50{ }^{\circ} \mathrm{C}$. With data obtained from the experimental part, the sizing of the

1 Escuela Superior Politécnica de Chimborazo, Facultad de Ciencias. Riobamba, Ecuador. gaby.26.94@hotmail.com

2 Escuela Superior Politécnica de Chimborazo, Facultad de Ciencias. Riobamba, Ecuador. sonia.vallejo@espoch.edu.ec

3 Escuela Superior Politécnica de Chimborazo, Facultad de Ciencias. Riobamba, Ecuador. marlene.garcía@espoch.edu.ec

4 Escuela Superior Politécnica de Chimborazo, Facultad de Ciencias. Riobamba, Ecuador. ecaluna2010@hotmail.com 
equipment was carried out for a production of $129.06 \mathrm{~kg}$ from pectin, using as feed 900 $\mathrm{kg}$ of potato husk; A financial study was conducted where the following values were obtained: IRR 94\% and VAN \$ 684.276,0. Validating the design of the process the characteristics from extracted pectin were obtained with the following values: $78.21 \%$ galacturonic acid, $7.93 \%$ humidity , $11.2 \%$ methyl's content and $1.49 \%$ ashes, these values compared with the ranges established by International Pectin Products Association IPPA for purity pectin, which are found within of ranges and therefore pectin obtained is considered acceptable.

Keywords: Pectin, Acid hydrolysis, Precipitation, Drying, Potato shells (solanum tuberosum), Galacturonic acid, Humidity.

\section{Resumen.}

La importancia de aprovechar los residuos orgánicos generados por la actividad doméstica alimenticia en los hogares, centros comerciales (restaurantes y mercados) de la Ciudad de Riobamba como las cáscaras de papas, para obtener nuevos productos que pueda ser aprovechada en la industria alimenticia como aditivo químico, de esta manera se puede minimizar el impacto ambiental de los residuos. Por esta razón se diseñó el proceso para la obtención de pectina a partir de las cáscaras de papa (Solanum tuberosum) en el Laboratorio de Química Analítica de la Escuela Superior Politécnica de Chimborazo, para ello se realizaron varios procesos empezando por el pretratamiento de la materia prima a la cual se determinó las características fisicoquímicas con un análisis bromatológico donde se obtuvieron los siguientes valores: Fibra 4,37 \%, Humedad 83,72 \%, la materia prima utilizada fue de la (Variedad de papa - Superchola). Seguido, se realizó la hidrólisis ácida donde se utilizó un diseño experimental -Taguchi, el mismo que permitió interactuar las variables más importantes desde este proceso: $\mathrm{pH}$, temperatura y tiempo de hidrólisis encontrando de esta manera las mejores condiciones de extracción: $\mathrm{pH}$ 1,5, tiempo de extracción 60 min y temperatura de extracción $90^{\circ} \mathrm{C}$, con estas condiciones se obtuvo un rendimiento del 14,34\%.Para la precipitación de la pectina se utilizó etanol al $96^{\circ}$ con una relación del $40 \%$ para el líquido de hidrólisis y el secado que se realizó a una temperatura de $50{ }^{\circ} \mathrm{C}$. Con los datos obtenidos de la parte experimental se procedió a realizar el dimensionamiento de los equipos para una producción de 129,06 kg de pectina, utilizando como alimentación $900 \mathrm{~kg}$ de cáscaras de papa, se realizó un estudio financiero donde se obtuvieron los siguientes valores: TIR 94\% y VAN \$ 684.276,0. Para validar el diseño del proceso se realizó la caracterización de la pectina extraída, obteniendo los siguientes valores: ácido galacturónico 78,21 \%, humedad 7,93 \%, contenido de metoxilos $11,2 \%$ y cenizas $1,49 \%$, estos valores fueron comparados con los rangos establecidos para pureza de pectinas por el IPPA (International Pectin 
Producers Association), los mismos que se encuentran dentro de los rangos y por lo tanto se considera aceptable la pectina obtenida.

Palabras claves: Pectina, Hidrólisis ácida, Precipitación, Secado, Cáscaras de papa (Solanum tuberosum), Ácido galacturónico, Humedad.

\section{Introducción.}

La pectina es un polisacárido con un alto poder de gelificación, de alto uso industrial que se puede obtener de las cortezas de frutos y vegetales, en el Ecuador no existe una industria que genere este tipo de aditivos alimenticios, por la cual se realizan importaciones de diferentes países fabricantes de pectina, los principales productores son Dinamarca, Holanda, Estados Unidos, Canadá, México, Suiza y Alemania, para ser utilizado en las pequeñas industrias alimenticias del Ecuador.

Conociendo de la inexistencia en el país de industrias dedicadas a la obtención de pectinas y sobre todo de la importancia a nivel industrial, se ha planteado el diseño del proceso de la obtención de pectina a partir de las cáscaras de papa, basado en el análisis experimental de laboratorio.

Se considera de importancia aprovechar los residuos orgánicos generados por la actividad doméstica alimenticia en los hogares, centros comerciales (restaurantes y mercados) de la Ciudad de Riobamba como las cáscaras de papas, las mismas que contiene una gran cantidad de fibra $14,21 \%$ de la cual se puede obtener una cantidad apreciable de pectina, la misma que pueda ser aprovechada en la industria alimenticia como aditivo químico, de esta manera se puede minimizar el impacto ambiental de los residuos.(Quispe, 2014, p 25)

\section{Metodologia.}

Para el proceso de hidrólisis ácida se tomó en cuenta el siguiente diseño experimental.

\section{Diseño Experimental - Método Taguchi}

Tabla 1: Factores de la Hidrólisis ácida

\begin{tabular}{llccl}
\hline $\mathbf{N}$ & \multicolumn{1}{c}{ FACTORES } & INDICADOR & \multicolumn{2}{c}{ NIVELES } \\
\hline 1 & \multicolumn{1}{c}{$\mathrm{pH}$} & $\mathrm{H}^{+}$ & $\mathrm{A} 1=1,5$ & $\mathrm{~A} 2=3$ \\
& Temperatura de & $\stackrel{\circ}{ } \mathrm{C}$ & $\mathrm{B} 1=90$ & $\mathrm{~B} 2=80$ \\
2 & Hidrólisis & $\mathrm{min}$ & $\mathrm{C} 1=60$ & $\mathrm{C} 2=45$ \\
3 & Tiempo de Hidrólisis & &
\end{tabular}

Como el método Taguchi explica el diseño experimental para este proyecto será $2^{3}$ debido a que posee 3 factores indicadores y dos niveles por lo tanto se deben realizar 8 experimentos, pero con la ayuda de un Software Minitab (confianza que normalmente es del 95\% con 5\% 
ISSN: 2602-8085

de probabilidad de error) se redujeron el número de experimentos para mejorar la eficiencia del proceso, y de igual forma simplificar esfuerzos de diseño estadístico.

Tabla 2: Condiciones de operación de la hidrólisis ácida

\begin{tabular}{|c|c|c|c|c|}
\hline & Muestra 1 & Muestra 2 & Muestra 3 & Muestra 4 \\
\hline \multirow{4}{*}{ CONDICIONES } & Acido: $\mathrm{HCl}$ & Acido: $\mathrm{HCl}$ & Acido: $\mathrm{HCl}$ & Acido: $\mathrm{HCl}$ \\
\hline & pH: 1,5 & pH: 1,5 & pH: 3 & pH: 3 \\
\hline & $\begin{array}{l}\text { Temperatura: } \\
90^{\circ} \mathrm{C}\end{array}$ & $\begin{array}{l}\text { Temperatura: } \\
80^{\circ} \mathrm{C}\end{array}$ & $\begin{array}{l}\text { Temperatura: } \\
90^{\circ} \mathrm{C}\end{array}$ & $\begin{array}{l}\text { Temperatura: } \\
80^{\circ} \mathrm{C}\end{array}$ \\
\hline & $\begin{array}{l}\text { Tiempo de } \\
\text { extracción: } \\
60 \text { min }\end{array}$ & $\begin{array}{l}\text { Tiempo de } \\
\text { extracción: } \\
45 \text { min }\end{array}$ & $\begin{array}{l}\text { Tiempo de } \\
\text { extracción: } \\
45 \text { min }\end{array}$ & $\begin{array}{l}\text { Tiempo de } \\
\text { extracción: } \\
60 \text { min }\end{array}$ \\
\hline
\end{tabular}

Tabla 3: Proceso de Obtención de la Pectina en el Laboratorio

\begin{tabular}{|c|c|}
\hline Proceso & Descripción \\
\hline $\begin{array}{l}\text { Inactivación } \\
\text { Enzimática }\end{array}$ & $\begin{array}{l}\text { La inactivación enzimática se realiza con el fin de mejorar el proceso de } \\
\text { extracción, y sobre todo inhibir la acción de las enzimas pépticas } \\
\text { presentes, el material seleccionado se calentó hasta alcanzar la } \\
\text { temperatura de } 100^{\circ} \mathrm{C} \text {, quedando el material listo para la hidrólisis acida. } \\
\text { Por cada } 100 \mathrm{~g} \text { de materia se agrega } 200 \mathrm{ml} \text { de agua. }\end{array}$ \\
\hline Hidrólisis Ácida & $\begin{array}{l}\text { Se utilizó un vaso de precipitación, tomando en cuenta la relación de agua } \\
\text { con la materia (1/25), el agua debe estar acidulada por tal motivo se utilizó } \\
\mathrm{HCl} \text {, hasta obtener el pH requerido } 1,5 \text {. El agua debe estar caliente hasta } \\
\text { obtener el valor de } 90^{\circ} \mathrm{C} \text {, al mismo tiempo se va agregando la muestra y } \\
\text { con una agitación constante para que no se deposite en el fondo, el tiempo } \\
\text { de hidrólisis es de } 60 \text { min. }\end{array}$ \\
\hline Filtrado 1 & $\begin{array}{l}\text { El filtrado es necesario para poder separar la materia sólida del líquido en } \\
\text { donde se encuentra la pectina, se realizó con una tela de lienzo. }\end{array}$ \\
\hline Concentración & $\begin{array}{l}\text { Se realiza la concentración hasta el } 50 \% \text { del líquido de hidrólisis filtrado a } \\
\text { la misma temperatura de extracción. }\end{array}$ \\
\hline Precipitado & $\begin{array}{l}\text { A la solución de hidrólisis se agregó etanol }\left(96^{\circ}\right) \text { lentamente y con } \\
\text { agitación contante durante } 5 \text { minutos (tomando en cuenta que en la etapa } \\
\text { de precipitado se utiliza el } 40 \% \text { ). }\end{array}$ \\
\hline
\end{tabular}




\begin{tabular}{|c|l|}
\hline Filtrado 2 & $\begin{array}{l}\text { Se realizó con un embudo en el cual se coloca en la parte superior papel } \\
\text { filtro de mayor porosidad, esto permite la separación por acción de la } \\
\text { gravedad de la concentración de etanol y pectina (quedando está en la } \\
\text { parte superior del papel filtro). }\end{array}$ \\
\hline Secado & $\begin{array}{l}\text { La pectina obtenida en el papel filtro se secó en una estufa a } 50 \text { oc por } 12 \\
\text { horas, se pesó para obtener el rendimiento de la pectina obtenida. }\end{array}$ \\
\hline Molienda & $\begin{array}{l}\text { Se realizó la molienda de la pectina para obtener un polvo más fino, y } \\
\text { esta pueda disolverse así su poder gelificante y capacidad de solubilidad } \\
\text { no son limitados por el tamaño de las partículas. }\end{array}$ \\
\hline Empacado & $\begin{array}{l}\text { Finalmente, la pectina obtenida fue empaquetada en bolsas de polietileno } \\
\text { herméticas, para su análisis posterior. }\end{array}$ \\
\hline
\end{tabular}

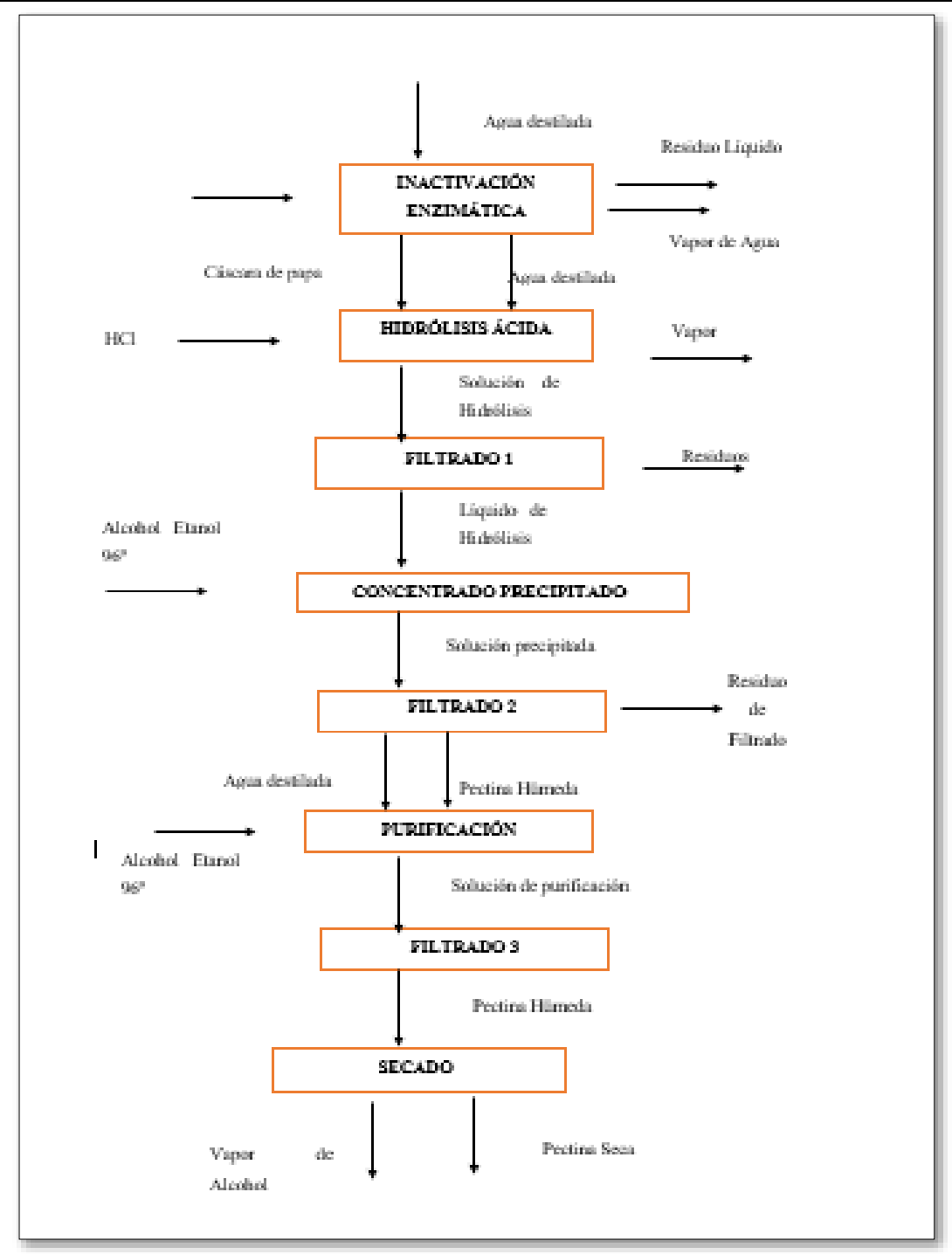




\section{Resultados.}

Caracterización de la materia prima (Cáscaras de papa)

Tabla 4: Análisis Bromatológico de la materia prima

\begin{tabular}{|l|l|l|}
\hline Componentes & Método & $\begin{array}{l}\text { Cáscara de Papa } \\
\text { (Base Húmeda) }\end{array}$ \\
\hline Cenizas \% & INEN 544 & 1,28 \\
\hline Proteína \% & INEN 543 & 2,05 \\
\hline Humedad \% & INEN 540 & 83,72 \\
\hline Fibra \% & INEN 522 & 4,37 \\
\hline Carbohidratos \% & - & 5,69 \\
\hline
\end{tabular}

Fuente: Laboratorio SAQMIC

Se realizó la caracterización de la materia prima con un análisis proximal donde se obtuvo los siguientes valores descritos en la Tabla estos parámetros corresponden a la variedad de papa Superchola.

\section{Obtención de la pectina a Escala Laboratorio}

Tabla 5: Obtención de la Pectina a Escala Laboratorio

\begin{tabular}{|l|l|}
\hline Muestra 1 & \\
CONDICIONES & \\
Acido: $\mathrm{HCl}$ & \\
Tem: 1,54 & \\
Tiempo de & \\
extracción: $60 \mathrm{~min}$ & \\
\end{tabular}

Para la obtención de la pectina en el laboratorio se utilizó el método estadístico Taguchi mediante el cual se pudo interactuar las siguientes variables: $\mathrm{pH}$, tiempo y temperatura de extracción, de los 4 experimentos realizados se hizo un balance de masa para determinar la mayor cantidad de pectina seca, se eligió la Muestra 1 de la misma se obtuvo 1,44 g. Entonces 
se establecieron las mejores condiciones de extracción de la pectina de las cáscaras de papa descritas en la Tabla 4 de la misma se obtuvo un porcentaje de rendimiento del 14,34\%.

\section{Caracterización de la Pectina Obtenida}

Tabla 6: Datos caracterización de la Pectina

\begin{tabular}{|c|c|}
\hline & $\begin{array}{c}\text { Pectina obtenida a nivel } \\
\text { Laboratorio }\end{array}$ \\
\hline Condiciones & $\begin{array}{c}\text { Acido: } \mathrm{HCl} \\
\text { pH: } 1,54 \\
\text { Temperatura: } 90^{\circ} \mathrm{C} \\
\text { Tiempo de extracción: } 60 \\
\text { min } \\
\text { Muestra } 1\end{array}$ \\
\hline $\begin{array}{c}\text { Rendimiento } \\
\%\end{array}$ & 14,34 \\
\hline $\begin{array}{c}\text { Humedad } \\
\%\end{array}$ & 7,93 \\
\hline $\begin{array}{c}\text { Cenizas } \\
\%\end{array}$ & 1,49 \\
\hline Acidez Libre & 0,612 \\
\hline Peso Equivalente & 1634 \\
\hline $\begin{array}{c}\text { Contenido de Metoxilos } \\
\%\end{array}$ & 11,2 \\
\hline $\begin{array}{c}\text { Contenido de Ácido } \\
\text { Galacturónico } \\
\%\end{array}$ & 78,21 \\
\hline $\begin{array}{c}\text { Grado de Esterificación } \\
\qquad \%\end{array}$ & 81,3 \\
\hline
\end{tabular}

Fuente: Laboratorio LACONAL 
En la caracterización de la pectina se obtuvieron los siguientes valores descritos en la Tabla 6 los mismos que según los organismos de control de pureza de pectina descritos en la tabla 7 están dentro de los rangos establecidos, por lo tanto, la pectina extraída es de alto metoxilo lo cual requiere de una cantidad superior del $60 \%$ de azúcar para poder gelificar.

Tabla 7: Organismo reguladores de pureza de pectina

\begin{tabular}{|l|l|l|}
\hline & $\begin{array}{l}\text { Especificaciones } \\
\text { USP }\end{array}$ & $\begin{array}{l}\text { Especificaciones } \\
\text { IPPA }\end{array}$ \\
\hline Humedad & $\max 10 \%$ & $\max 12 \%$ \\
\hline Cenizas & -- & $\max 1,5 \%$ \\
\hline $\begin{array}{l}\text { Contenido de } \\
\text { Metoxilos }\end{array}$ & $\min 6,7 \%$ & -- \\
\hline $\begin{array}{l}\text { Contenido de } \\
\text { Ácido } \\
\text { Galacturónico }\end{array}$ & $\min 74 \%$ & $\min 65 \%$ \\
\hline
\end{tabular}

Fuente: (Herbstreith\&fox, 2018)

\section{Análisis de Espectro Infrarrojo}

Los análisis de Espectro Infrarrojo se realizaron en el Laboratorio de Análisis Instrumental de la ESPOCH.

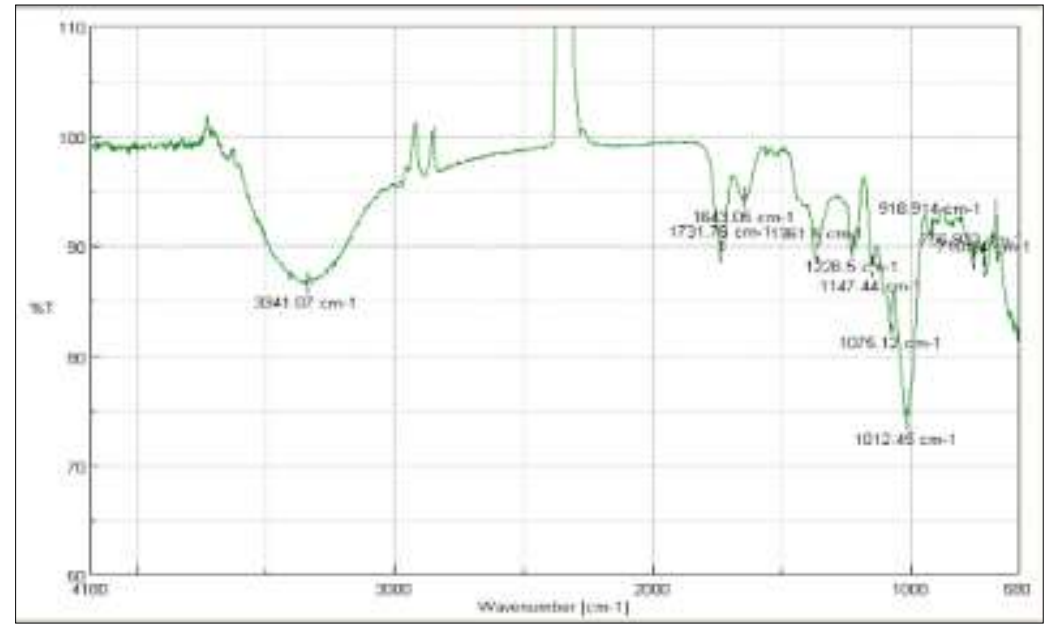

Figura 2: Espectro Infrarrojo de la pectina obtenida

Fuente: Laboratorio Análisis Instrumental-ESPOCH 


\section{(1) Ciencia Digital}

En la Figura 2 claramente se evidencia la presencia de los grupos funcionales característicos: $1731,76 \mathrm{~cm}^{-1}$ se encuentra la banda $\mathrm{C}=\mathrm{O}$ del éster, $1643,05 \mathrm{~cm}^{-1}$ la banda de $\mathrm{C}=\mathrm{O}$ del ácido, 1361,5 $\mathrm{cm}^{-1}$ la banda de C-H, entre $\mathrm{cm}^{-1}$ la banda entre 1226,5-1012,45 de $\mathrm{cm}^{-1} \mathrm{C}$ $\mathrm{O}$ y por último la banda de $-\mathrm{OH}$ se encuentra en $3341,07 \mathrm{~cm}^{-1}$

\section{Dimensionamiento de los equipos a escala industrial para la obtención de pectina}

Los equipos necesarios para la obtención de pectina se muestran en la Figura la misma que procesara $900 \mathrm{~kg}$ de cáscaras de papa, para obtener $129 \mathrm{~kg}$ de pectina al día.

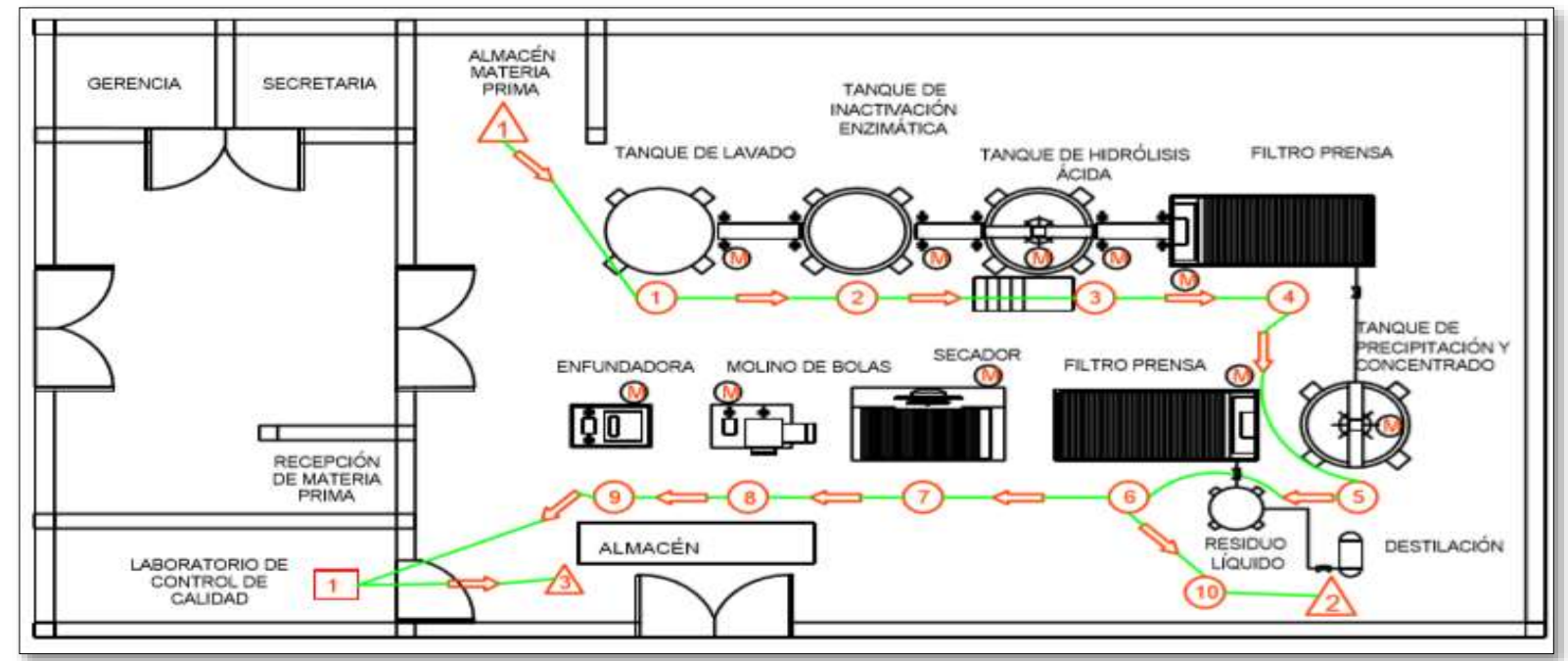

Figura 3: Distribución de la Planta procesadora de pectina

\section{Presupuesto y Viabilidad del Proyecto}

Tabla 8: Estudio Financiero

\begin{tabular}{|c|c|c|c|}
\hline TASA DE RENDIMIENTO DEL MERCADO & $12,00 \%$ & Periodo anterior al cambio de signo & 0 \\
\hline VAN & $\$ 684.276,0$ & \multicolumn{2}{|l|}{ Valor absoluto del flujo acumulado } \\
\hline TIR & $94 \%$ & Flujo de caja en siguiente periodo & 163039,44 \\
\hline & & Periodo de playback & 0,977309539 \\
\hline & & & \\
\hline & & 1,97 & Años se recupera lo invertido \\
\hline Suma Ingresos & $\$ 1.619 .241,79$ & & \\
\hline Suma Egresos & $\$ 1.094 .305,79$ & & \\
\hline Costos de Inversión & $\$ 934.965,79$ & & \\
\hline Relación Costo Beneficio & 1,731872773 & & \\
\hline
\end{tabular}


Se calcularon los indicadores financieros más importantes que son el: TIR (Tasa Interna de Retorno) 94\% y VAN (Valor Actual Neto) \$ 684.276,0 para poder determinar que el proyecto es económicamente viable, la relación costo beneficio será de \$ 0,73 por cada dólar que se invierta y nuestro periodo de recuperación de lo invertido será 1,93 años.

Se determinó además el precio al público será de $\$ 13,81$ por cada kilogramo, lo cual nos permite determinar que es el $58,76 \%$ menos del precio que se comercializa en la actualidad $\$ 23,50$ por cada kilogramo.

\section{Conclusiones y Recomendaciones}

- Se validó el diseño de ingeniería con la caracterización de la pectina obteniendo los siguientes valores: ácido galacturónico 78,21 \%, humedad 7,93\%, contenido de metoxilos $11,2 \%$ y cenizas $1,49 \%$, los mismo que cumplen con los rangos que el IPPA (International Pectin Producers Association) propone para determinar la pureza de una pectina.

- Es importante tomar en cuenta la agitación en el proceso de hidrólisis ácida, de tal forma la materia vegetal no quedará en el fondo del recipiente.

- Para realizar la inactivación enzimática debe asegurarse que se llegue a la temperatura de ebullición del agua, así se podrá inhibir la actividad de las enzimas pectinasas y será más eficiente el proceso de extracción.

- Para reducir la cantidad de etanol a utilizar a nivel industrial se necesita concentrar la solución hasta el 50\%, la misma no afecta en nada el rendimiento final.

\section{Referencias bibliográficas.}

Álvarez, E. (2007). Desarrollo de un proceso a escala laboratorio para la obtención de pectina y taninos a partir de la algarroba (Hymenaea Courbaril-L), para ser utilizados en la industria alimenticia y la del cuero, respectivamente [En linea],(Tesis), (Pregrado,)pp. 29-28 Disponible en : https://repository.eafit.edu.co/bitstream/handle/10784/342/ErikaMaria_Alvarez Ramirez_2007.PDF?sequence=1. Consultado en: 2018-10-18].

Acos, T. y Cauja, L. (2017). "Diseño de un proceso para la obtención de detergente líquido mediante la extracción de saponina extraída de quinua (chenopodium quinoa willd), chocho (lupinus mutabilis sweet) y cabuya (sisalana perrine)" [en línea] ,(Tesis), (Pregrado) Escuela Superior Politécnica de Chimborazo. Facultad
de
Ciencias.pp.
29-28
Disponible
en: 
http://dspace.espoch.edu.ec/bitstream/123456789/6341/1/85T00415.pdf, Consultado en: 2018-09-15.

Barragán, J.C.A., Zerpa, S.A.I., Castillo, M.L.S., Haro, M.R., Alarcón, W.N. y Gasco, F.O. (2014). Efecto de la temperatura y pH sobre la actividad y estabilidad de pectinasas producidas por Bacillus spp. Revista Científica de la Facultad de Ciencias Biológicas, vol. 34, no. 1 ,pp. 33-41.

Bravo, M. (2015). " Comparación de la pectina obtenida a partir del aprovechamiento de las cáscaras de banano y cacao por el método de hidrólisis ácida ". [en línea] ,(Tesis), (Pregrado) Universidad de Guayaquil.pp. 19-25. Disponible en: http://repositorio.ug.edu.ec/bitstream/redug/8938/1/BCIEQ-T0113\%20Bravo\%20Mat\%C3\%ADas\%20Angela\%20Mabel\%3b\%20Condo\%2 0Franco\%20Evelyn\%20Ivonne.pdf, Consultado en: 2018-09-22

Cabarcas Henao, E., Guerra Benedetti, A.F. y Henao Balseiro, C.A. (2012). Extracción Y Caracterización De Pectina Apartir De Cáscaras De Plátano Para Desarrollar Un Diseño General Del Proceso [en línea], pp. 71. ISSN 1098-6596. Disponible en: DOI 10.1017/CBO9781107415324.004. Consultado en: 2018-0915.

Cantery, M.H.G. (2012). Pectina : da Matéria-Prima ao Produto Final. Revista Artigo , vol. 22, pp. 149-157.

Center, I.P. (2015). Procesamiento y Usos de la Papa. [en línea]. Disponible en: https://cipotato.org/es/lapapa/procesamiento-y-usos-de-la-papa/.Consultado en: 2018-09-22.

De Pablo Busto, M. y Moragas Ecuentra, M. (2017).Recopilación normas microbiológicas de los alimentos y asimilados (superficies, aguas diferentes de consumo, aire, subproductos) otros parámetros fiscoquímicos de interés sanitario. [en línea] ,(Tesis), (Pregrado) pp. 8-10. Disponible en: eurocarne.com/daal/al/informes/a2/normas-microbiologicas.pdf. Consultado en: 2018-09-08.

HerbstreithFox. (2018). The Specialists for Pectin.Disponible en: http://www.herbstreith-fox.de/. Consultado el: 2018-09-1

Quispe, J. (2014). Obtención y caracterización de la fibra dietética a partir de los residuos de papa (Solanum tuberosum). [en línea] ,(Tesis), (Pregrado) .Universidad Nacional de Huancavelica. pp. 62-66 .Disponible en: repositorio.unh.edu.pe/handle/UNH/733\%0A. Consultado el : 2018-09-07 
PARA CITAR EL ARTÍCULO INDEXADO.

Toapanta, E. G., Vallejo Abarca, S., García, M., \& Caluña Sánchez, E. (2019). Diseño de un proceso para la obtención de pectina en medio ácido a partir de la cáscara de papa (Solanum tuberosum). Ciencia Digital, 3(2.6), 115-126. https://doi.org/10.33262/cienciadigital.v3i2.6.522

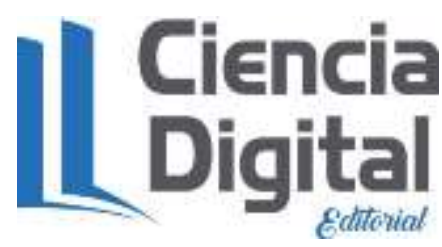

El artículo que se publica es de exclusiva responsabilidad de los autores y no necesariamente reflejan el pensamiento de la Revista Explorador Digital.

El articulo queda en propiedad de la revista y, por tanto, su publicación parcial y/o total en otro medio tiene que ser autorizado por el director o editor de la Revista Explorador Digital.
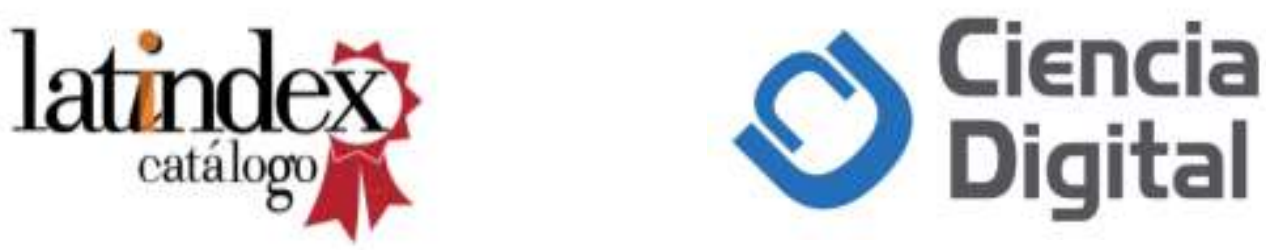